\title{
Skeletal Muscle Extracellular Matrix: Composition, Structure and Function
}

\author{
Created by: Khurshid Ahmad $^{1}$, Inho Choi ${ }^{2}$, Yong-ho Lee $^{3}$ \\ 1, Department of Medical Biotechnology, Yeungnam University, Gyeongsan-38541, Republic \\ of Korea; ahmadkhursheed2008@gmail.com \\ 2, Department of Medical Biotechnology, Yeungnam University, Gyeongsan 38541, South \\ Korea; inhochoi@ynu.ac.kr \\ 3, Department of Biomedical Sciences, Daegu Catholic University, Gyeongsan 38430, \\ Korea; ylee325@cu.ac.kr
}

Version received: 17 June 2020

check for

updates

The skeletal muscle provides movement and support to the skeleton, controls body temperature, and regulates the glucose level within the body. This is the core tissue of insulin-mediated glucose uptake via glucose transporter type 4 (GLUT4). The extracellular matrix (ECM) provides a scaffold for cells, controlling biological processes, and providing structural as well as mechanical support to surrounding cells. Disruption of ECM homeostasis results in several pathological conditions. Various ECM components are typically found to be augmented in the skeletal muscle of obese and/or diabetic humans. A better understanding of the importance of skeletal muscle ECM remodeling, integrin signaling, and other factors that regulate insulin activity may help in the development of novel therapeutics for managing diabetes and other metabolic disorders.

\section{Introduction}

The skeletal muscle constitutes nearly $40 \%$ of body mass and is primarily composed of myofiber, multinucleated contractile cells, and mainly provides mobility, protects and supports the skeleton, and regulates the body temperature and glucose homeostasis within the body. The extracellular matrix (ECM) is composed of structural glycoproteins like collagens, laminin (s), and fibronectin (FN) bound to proteoglycans (PGs), which all help to maintain skeletal muscle integrity and provides constructional support. Collagen is the key component of fibrous protein in SM ECM, accounting for up to $10 \%$ of SM by weight and forming a network of intramuscular connective tissue (IMCT) ${ }^{[1]}$. IMCT is organized into endomysium (inner), perimysium, and epimysium (outer) layers. Collagen I and III are abundant in the IMCT, while other types of collagens are also present [2].

Collagen I is the main component of epimysium and perimysium, while collagen III is a minor component. Both, collagen I and III are the major components of the endomysium [3]. The endomysium shares a boundary with the myofiber sarcolemma at the basal lamina of the basement membrane (BM). The BM is composed of the reticular lamina and basal lamina, which contain collagen IV, nidogen, perlecan, and laminins [4]. Collagen VI, XV, and XVIII are also components of SM BM [5]. Laminins are the key components of the SM BM surrounding muscle fibers and stimulate proliferation, migration, differentiation, and survival of myoblasts [6]. Laminin binds with its transmembrane receptors dystroglycan and integrin $\alpha 7 \beta 1$ in the SM. There are several isoforms of laminins, and among them, laminin211 is the most abundant isoform found in the adult SM BM [7][8].

\section{Collagen}

Collagens are the most abundant protein in multicellular organisms and are the major component of the ECM 9 . The collagen gene family consists of 46 genes encoding 28 different types of proteins comprising different combinations; each type of collagen protein consists of homo- and hetero-trimers made of three polypeptide chains ${ }^{[10]}$. Collagens are the most prominent ECM component in SM as they play a crucial role in the regulation of cell attachment and differentiation, providing elasticity and tensile strength to bones [11]. There are 28 diverse forms of collagen, of which 11 types (collagen I, III, IV, V, VI, XII, XIII, XIV, XV, XVIII, and XXII) have been identified in mature SM [11]12]. Collagen I, III, V, IX, and XI are the fibrillar collagens found in SM, with I and III types being the most abundant, accounting for almost $75 \%$ of total SM collagen [2]. Collagen IV, a triple-helical molecule, is the key structural component of the basal lamina. Both fibrogenic and myogenic cells are known to secrete collagen IV [13]. 
Collagen type I alpha 1 and 2 chains (COL1A1/2) are fibrillar collagens found in all the three (endo-, peri-, and epimysium) layers; COL1A1/2 forms parallel fibers and determines tensile strength and rigidity in SM. Collagen type III alpha 1 chain (COL3A1) is also a fibrillar collagen that appears in endo- and perimysium as well as the myotendinous junction and forms a loose meshwork of fibers [14]. Collagen type $\mathrm{V}$ alpha 1-3 chains (COL5A1-3) controls fibrillogenesis [14]. Collagen type $\mathrm{VI}$ alpha $1-6$ chains (COL6A1-6) are the main beaded filaments: the $\alpha 6$ chain is predominantly found in endo- and perimysium; the $\alpha 3$ chain in basal lamina; and the $\alpha 5$ chain in the myotendinous junction (MTJ) ${ }^{[15]}$. Collagen $\mathrm{VI}$ is known to interact with several ECM components and cell surface receptors, and has a significant role in maintaining the functional integrity of the SM. Mutations in the COL6A1, COL6A2, and COL6A3 genes are associated with muscle disorders namely Ullrich congenital muscular dystrophy and Bethlem myopathy 16$]$. Collagen type XV alpha 1 chain (COL15A1), collagen type XVIII alpha 1 chain (COL18A1), and collagen type XIX alpha 1 chain (COL19A1) are components of the BM of SM [12][17]. Collagen type XXII alpha 1 chain (COL22A1) is found in the MTJ and assimilates ECM components, providing mechanical stability to the MTJ. The knockdown of the COL22A1 gene results in muscular dystrophy in zebrafish by disruption of the MTJ 18$][19]$.

Generally, collagen is produced by fibroblasts in mature SM, but in fibrosis conditions, it may be produced by other cell types, such as myofibers, MSCs, inflammatory cells, or endothelial cells. Fibrosis is the aggregation of excess ECM components, common in most myopathies. Fibrosis in SM occurs during myopathy, aging, and diabetes, usually characterized by increased endomysium as well as perimysium [1]. Expression of collagen I, III, and IV is reported to be increased throughout diet-induced insulin resistance (IR) $[\underline{20}$. Both the murine model and human patients show that the level of SM collagen is higher in insulin resistance (IR) [21]. In a comparative study, total collagen content was observed to increase in obese insulin-resistant individuals compared with lean individuals [22]. Diabetes-induced alterations in SM concern the structure of the BM and the actions of the enzymes responsible for the synthesis of collagen. Gene expression of several collagen types (I, III, IV, V, VI, and XV) was found to be reduced in streptozotocin-induced diabetic mice in a microarray analysis of SM transcriptome. Additionally, mRNA expression of several non-collagenous proteoglycans and glycoproteins was found to be increased in diabetic muscles [23].

\section{Laminin}

Laminin is a heterotrimeric glycoprotein and a foremost component of the BM. Laminin-211 (previously named merosin) is the most abundant isoform of laminin in the BM of adult SM. However, other isoforms exist during myogenesis and at junctional regions (e.g., the neuromuscular junction and the myotendinous junction) of the muscle fiber [24]. Laminin-211 is composed of one $\alpha 2$ chain, one $\beta 1$ chain, and one $\gamma 1$ chain. The biological functions of the laminins are typically reliant on binding to the cell surface receptors. Two main groups of laminins receptors are known (i.e., integrins and non-integrins).

Integrin $\alpha 7 \beta 1$ has been recognized as the main receptor for laminins in adult SM. The $\alpha 7$ subunit is present as $\alpha 7 \mathrm{~A}$ and $B$ in adult SM of mouse and human, where it attaches with an integrin $\beta 1$ splice form ( $\beta 1 D$ subunit) 25$]$. Integrin $\alpha 7 B \beta 1 D$ is expressed throughout the sarcolemma, while $\alpha 7 A \beta 1 D$ expression is limited to junctional sarcolemma [26]. The foremost non-integrin cell surface receptor of laminins in SM is dystroglycan, a central piece of the dystrophinglycoprotein complex (DGC) [27]. Laminin-211 binds to dystroglycan through O-linked mannose chains of $\alpha$ dystroglycan ( $\alpha-D G$ ). $\alpha-D G$ is non-covalently connected to $\beta$-dystroglycan, which binds to dystrophin inside the muscle fiber. Other non-integrin receptors of laminin-211 in SM includes sulfatides and syndecans [28][29]. Laminin-211 also interacts with several other ECM components, for example perlecan, agrin, and nidogen [24].

\section{ECM receptors}

\section{Integrin}

Integrins are the key receptors of most SM ECM components, facilitating mechanical communication between ECM components and cells; they have several critical roles, such as cell attachment, migration, and differentiation [30]. Integrins also facilitate unique bidirectional signaling between ECM and intracellular molecules ("inside-out" and "outside-in" signaling) [31]. Integrins are heterodimeric, having two subunits ( $\alpha$ and $\beta$ ). SM express seven $\alpha$ subunits $(\alpha 1, \alpha 3, \alpha 4, \alpha 5, \alpha 6, \alpha 7$, and $\alpha v)$ in combination with the $\beta 1$ subunit [32][33]. 
Collagen and laminin in SM bind mostly with the $\beta 1$ subunit of integrin 34$]$. In vertebrates, collagen binds with four integrin receptors that have $\beta 1$-subunits in association with any of the $\alpha 1-, \alpha 2-, \alpha 10-$, or $\alpha 11$-subunits [35]. Previously it was observed a reduced activation of focal adhesion kinase (FAK) in insulin-resistant SM of high-fat fed rats that specify the role for integrin-collagen interaction in the expansion of $I R[36]$. Mice with integrin $\beta 1$ subunit-deficient striated muscle show IR, as measured by diminishing insulin-mediated glucose uptake and glycogen synthesis in SM and indicated by a reduction in phosphorylation of protein kinase B (AKT) Ser-473 [37]. Downstream signaling of integrins depends on the involvement of the intracellular kinases, FAK and integrin-linked kinase (ILK). Disrupted signaling of integrin and the subsequent inflection of FAK and ILK are found to regulate insulin sensitivity in SM, possibly via altered capillary density [21][38]. As the muscle capillaries are established in direct contact with the ECM, any defect in the recruitment of these capillaries leads to development of $S M I R[39$. Zong et al. suggested a connection between abnormal signaling of integrin and the development of T2DM. They observed a decrease of insulin-mediated glucose infusion rate and clearance of glucose in the muscle-specific integrin $\beta 1$-deficient mice, notwithstanding any changes in the intake of food, weight, glucose (fasting), GLUT4 expression, or insulin levels [40]. Meakin et al. reported that $\beta 2$-integrins control homeostasis of glucose during high-fat feeding, mostly through actions on SM, to affect the metabolic phenotype in vivo ${ }^{[41]}$. Furthermore, in an experiment with obese high-fat fed mice, with deletion of $\alpha 2$ integrin from the whole body, a moderate reverse of diet-induced muscle IR was observed, as confirmed by augmented insulin-mediated uptake of glucose through a hyperinsulinemic-euglycemic clamp, and increased insulin signaling [42].

\section{Non-integrin ECM receptors}

\section{CD44}

CD44 is a glycoprotein cell surface receptor for a number of ECM components, such as hyaluronan (HA), osteopontin, collagen I, and fibronectin, which are mostly present in the adipose tissue, SM, pancreas, liver, and endothelium. CD44 plays a significant role in the regulation of diverse cellular functions, including cell aggregation, endothelial cell proliferation, and immune cell migration and activation [43]. A genome-wide association study demonstrated that the cd44 gene is associated with the pathogenesis of T2DM[44]. Hasib et al. hypothesized that high-fat feeding in mice increases HA content and expression of CD44; this activates CD44 signaling and enables muscle capillary rarefaction, successively leading to the development of IR. Interruption of this pathway at several stages, for example, by reduction of HA via PEGPH20 treatment and/or CD44 deletion, helps in reducing IR in SM. Hasib et al. concluded that HA-CD44 signaling might be a probable therapeutic target for IR and T2DM [45].

\section{Dystroglycan}

Dystroglycan, a transmembrane protein comprising $\alpha$ and $\beta$ subunits, is a constituent of the dystroglycan complex (DGC) that enables an interaction between the ECM and cytoskeleton of muscle cells. The DGC is a key receptor system for the components of the ECM in SM [46]. Dystroglycan confers stability to myotube by binding to its ligand (laminin-211) during muscle contraction [47]. As dystroglycan is a heavily glycosylated protein, any defect in this glycosylation may lead to several forms of muscular dystrophy [47]. The DGC is involved in essential cell-signaling processes and serves as a binding platform for several ligands, including nitric oxide synthase, which is known to stimulate the transport of glucose. Thus, disruption in the DGC or its components may lead to abnormal insulin signaling in SM fibers and result in altered functionality. Collectively, alterations in the DGC may create changes in glucose metabolism; for example, IR in the SM of patients with muscular dystrophy [48][49].

\section{Syndecans}

Syndecans are transmembrane proteoglycans that form a core set of proteins linked to linear carbohydrate chains, known as glycosaminoglycans (GAG) [50]51]. In addition to binding growth factors through the glycosaminoglycan chains, Syndecans can bind directly to ECM molecules ${ }^{[52]}$.

\section{Muscle stem cells and Extracellular Matrix}

Muscle fibers or myofibers are the functional units of skeletal muscles and are formed during embryogenesis when 
myoblasts fuse to form myotubes. Under normal conditions, MSCs are usually in the quiescent phase and remain in this phase until they are invoked by injury and exercise. Small injuries may initiate minimal MSC proliferation, whereas major injuries can result in the recruitment of large numbers of MSCs, and subsequent proliferation before differentiation [53]. Many factors regulate MSC activation, some of the more widely explored factors include muscle regulatory factors (MRFs, such as, MYF5, MYOD, and myogenin), hepatocyte growth factor (HGF), and neuronal nitric oxide synthase (NOS) [54]. MSCs are positioned between the sarcolemma (cell membrane) and the basal membrane (basal lamina; BL) of muscle fibers, and the balance between quiescent and activated forms of MSCs is usually sustained in this niche [55]. The ability to regenerate skeletal muscle is primarily dependent upon the interaction between MSCs and their microenvironment. BL is covered by an ECM network, which directly contacts MSCs. The main components of $\mathrm{BL}$ are type IV collagen and laminin-2, and their concentrations are muscle fiber type dependent. Other components that comprise BL include collagen VI, perlecan, nidogen, FN and other glycoproteins and PGs [56][57].

\section{References}

1. Allison R. Gillies; Richard L. Lieber; Structure and function of the skeletal muscle extracellular matrix. Muscle \& Nerve 2011, 44, 318331, 10.1002/mus.22094.

2. Turney J. McKee; George Perlman; Martin Morris; Svetlana V. Komarova; Extracellular matrix composition of connective tissues: a systematic review and meta-analysis. Scientific Reports 2019, 9, 10542, 10.1038/s41598-019-46896-0.

3. N Light; A E Champion; E R Savolainen; D Brocks; L Ala-Kokko; K I Kivirikko; W A Galloway; G Murphy; J D Sandy; J Gavrilovic; et al.T E CawstonJ J ReynoldsA J BaileyT J SimsA A ChiodoD O SillenceW G ColeJ F Bateman Characterization of muscle epimysium, perimysium and endomysium collagens. Biochemical Journal 1984, 219, 1017-1026, 10.1042/bj2191017.

4. LeBleu, V.S.; Macdonald, B.; Kalluri, R; Structure and Function of Basement Membrane. Exp Biol Med (Maywood) 2007, 232, 11211129.

5. Willi Halfter; Sucai Dong; Barbara Schurer; Gregory J. Cole; Collagen XVIII Is a Basement Membrane Heparan Sulfate Proteoglycan. Journal of Biological Chemistry 1998, 273, 25404-25412, 10.1074/jbc.273.39.25404.

6. Jachinta E. Rooney; Praveen B. Gurpur; Zipora Yablonka-Reuveni; Dean J Burkin; Laminin-111 Restores Regenerative Capacity in a Mouse Model for $\alpha 7$ Integrin Congenital Myopathy. The American Journal of Pathology 2009, 174, 256-264, 10.2353/ajpath.2009.080522.

7. Takako Sasaki; Richard Giltay; Ulrika Talts; Rupert Timpl; Jan F. Talts; Expression and Distribution of Laminin $\alpha 1$ and $\alpha 2$ Chains in Embryonic and Adult Mouse Tissues: An Immunochemical Approach. Experimental Cell Research 2002, 275, 185-199, 10.1006/excr.2002.5499.

8. K. Ehrig; I. Leivo; W. S. Argraves; E. Ruoslahti; E. Engvall; Merosin, a tissue-specific basement membrane protein, is a laminin-like protein. Proceedings of the National Academy of Sciences 1990, 87, 3264-3268, 10.1073/pnas.87.9.3264.

9. Ricard-Blum, S; The collagen family. Cold Spring Harb Perspect Biol 2011, 3, a004978.

10. Janna K. Mouw; Guanqing Ou; Valerie M. Weaver; Extracellular matrix assembly: a multiscale deconstruction. Nature Reviews Molecular Cell Biology 2014, 15, 771-785, 10.1038/nrm3902.

11. Khurshid Ahmad; Sibhghatulla Shaikh; Syed Sayeed Ahmad; Eun Ju Lee; Inho Choi; Cross-Talk Between Extracellular Matrix and Skeletal Muscle: Implications for Myopathies. Frontiers in Pharmacology 2020, 11, 142, 10.3389/fphar.2020.00142.

12. Csapo, R.; Gumpenberger, M.; Wessner, B; Skeletal Muscle Extracellular Matrix - What Do We Know About Its Composition, Regulation, and Physiological Roles? A Narrative Review. Front Physiol 2020, 11, 253.

13. Uwe Kühl; Mücella Öcalan; Rupert Timpl; Richard Mayne; Elizabeth Hay; Klaus Von Der Mark; Role of muscle fibroblasts in the deposition of type-IV collagen in the basal lamina of myotubes. Differentiation 1984, 28, 164-172, 10.1111/j.1432-

0436.1984.tb00279.x.

14. Vuokko Kovanen; Intramuscular Extracellular Matrix: Complex Environment of Muscle Cells. Exercise and Sport Sciences Reviews 2002, 30, 20-25, 10.1097/00003677-200201000-00005.

15. Sabatelli, P.; Gualandi, F.; Gara, S.K.; Grumati, P.; Zamparelli, A.; Martoni, E.; Pellegrini, C.; Merlini, L.; Ferlini, A.; Bonaldo, P., et al; et al. Expression of collagen VI alpha5 and alpha6 chains in human muscle and in Duchenne muscular dystrophy-related muscle fibrosis. Matrix Biol 2012, 31, 187-196.

16. Bonnemann, C.G; The collagen VI-related myopathies: muscle meets its matrix. Nat Rev Neurol 2011, 7, 379-390.

17. Guillon, E.; Bretaud, S.; Ruggiero, F; Slow Muscle Precursors Lay Down a Collagen XV Matrix Fingerprint to Guide Motor Axon Navigation. J Neurosci 2016, 36, 2663-2676.

18. Manuel Koch; Joerg Schulze; Uwe Hansen; Todd Ashwodt; Uglas R. Keene; William J Brunken; Robert E. Burgeson; Peter Bruchner; Leena Bruckner-Tuderman; A novel marker of tissue junctions, collagen XXII. Journal of Biological Chemistry 2004, 279, 22514-21, 10.1074/jbc.M400536200.

19. B. Charvet; A. Guiraud; M. Malbouyres; D. Zwolanek; E. Guillon; S. Bretaud; Catherine Monnot; J. Schulze; H. L. Bader; B. Allard; et al.M. KochF. Ruggiero Knockdown of col22a1 gene in zebrafish induces a muscular dystrophy by disruption of the myotendinous 
junction. Development 2013, 140, 4602-4613, 10.1242/dev.096024.

20. Charmaine S. Tam; Jeffrey D. Covington; Sudip Bajpeyi; Yourka Tchoukalova; David Burk; Darcy L. Johannsen; Cristina M. Zingaretti; Saverio Cinti; Eric Ravussin; Weight gain reveals dramatic increases in skeletal muscle extracellular matrix remodeling.. The Journal of Clinical Endocrinology \& Metabolism 2014, 99, 1749-57, 10.1210/jc.2013-4381.

21. Kang, L.; Ayala, J.E.; Lee-Young, R.S.; Zhang, Z.; James, F.D.; Neufer, P.D.; Pozzi, A.; Zutter, M.M.; Wasserman, D.H; Diet-induced muscle insulin resistance is associated with extracellular matrix remodeling and interaction with integrin alpha2beta1 in mice. Diabetes 2011, 60, 416-426.

22. Rachele Berria; Lishan Wang; Dawn K. Richardson; Jean Finlayson; Renata Belfort; Thongchai Pratipanawatr; Elena A. De Filippis; Sangeeta Kashyap; Lawrence J. Mandarino; Increased collagen content in insulin-resistant skeletal muscle. American Journal of Physiology-Endocrinology and Metabolism 2006, 290, E560-E565, 10.1152/ajpendo.00202.2005.

23. T. Maarit Lehti; Mika Silvennoinen; Riikka Kivela; Heikki Kainulainen; Jyrki Komulainen; Maarit Lehti; Effects of streptozotocininduced diabetes and physical training on gene expression of extracellular matrix proteins in mouse skeletal muscle. American Journal of Physiology-Endocrinology and Metabolism 2006, 290, E900-E907, 10.1152/ajpendo.00444.2005.

24. Johan Holmberg; Madeleine Durbeej; Laminin-211 in skeletal muscle function. Cell Adhesion \& Migration 2013, 7, 111-121, 10.4161/cam.22618.

25. D J Burkin; S J Kaufman; The alpha7beta1 integrin in muscle development and disease. Cell and Tissue Research 1999, 296, 183190.

26. Ulrike Mayer; Integrins: Redundant or Important Players in Skeletal Muscle?. Journal of Biological Chemistry 2003, 278, 1458714590, 10.1074/jbc.r200022200.

27. James M. Ervasti; Kevin P. Campbell; Membrane organization of the dystrophin-glycoprotein complex. Cell 1991, 66, 1121-1131, 10.1016/0092-8674(91)90035-w.

28. Jan F. Talts; Zeynep Andac; Walter Göhring; Andrea Brancaccio; Rupert Timpl; Binding of the G domains of laminin alpha1 and alpha2 chains and perlecan to heparin, sulfatides, alpha-dystroglycan and several extracellular matrix proteins. The EMBO Journal 1999, 18, 863-870, 10.1093/emboj/18.4.863.

29. Nobuharu Suzuki; Fumiharu Yokoyama; Motoyoshi Nomizu; Functional Sites in the Laminin Alpha Chains. Connective Tissue Research 2005, 46, 142-152, 10.1080/03008200591008527.

30. Richard O. Hynes; Integrins: bidirectional, allosteric signaling machines. Cell 2002, 110, 673-687.

31. Khurshid Ahmad; Eun Ju Lee; Sibhghatulla Shaikh; Anuj Kumar; Kummara Madhusudana Rao; So-Yong Park; Jun O Jin; Sung Soo Han; Inho Choi; Ahmad Khurshid; et al.Ju Lee EunShaikh SibhghatullaKumar AnujMadhusudana Rao KummaraPark So-YongO Jin JunSoo Han Sunglnho Choi Targeting integrins for cancer management using nanotherapeutic approaches: Recent advances and challenges. Seminars in Cancer Biology 2019, -, -, 10.1016/j.semcancer.2019.08.030.

32. Gullberg, D.; Velling, T.; Lohikangas, L.; Tiger, C.F; Integrins During Muscle Development and in Muscular Dystrophies. Front Biosci 1998, 3, D1039-1050.

33. Williams, A.S.; Kang, L.; Wasserman, D.H; The extracellular matrix and insulin resistance. Trends in Endocrinology \& Metabolism 2015, 26, 357-366.

34. Ashley S. Williams; Li Kang; David H. Wasserman; The extracellular matrix and insulin resistance. Trends in Endocrinology \& Metabolism 2015, 26, 357-366, 10.1016/j.tem.2015.05.006.

35. McCall-Culbreath, K.D.; Zutter, M.M; Collagen receptor integrins: rising to the challenge. Curr Drug Targets 2008, 9, 139-149.

36. Bisht, B.; Goel, H.L.; Dey, C.S; Focal adhesion kinase regulates insulin resistance in skeletal muscle. Diabetologia 2007, 50, 10581069.

37. Zong, H.; Bastie, C.C.; Xu, J.; Fassler, R.; Campbell, K.P.; Kurland, I.J.; Pessin, J.E; Insulin resistance in striated muscle-specific integrin receptor beta1-deficient mice. J Biol Chem 2009, 284, 4679-4688.

38. Anne-Sofie Graae; Niels Grarup; Rasmus Ribel-Madsen; Sara H. Lystbæk; Trine Boesgaard; Harald Staiger; Andreas Fritsche; Niels Wellner; Karolina Sulek; Mads Kjolby; et al.Marie Balslev BackeSabina ChubanavaClara PratsAnnette K. SerupJesper B.

BirkJohanne DubailLinn GillbergSara G. VienbergAnders NykjærBente KiensJørgen F P WojtaszewskiSteen LarsenSuneel S. ApteHans-Ulrich HäringAllan VaagBjörn ZetheliusOluf PedersenJonas T. TreebakTorben HansenBirgitte Holst ADAMTS9 Regulates Skeletal Muscle Insulin Sensitivity Through Extracellular Matrix Alterations. Diabetes 2019, 68, 502-514, 10.2337/db18-0418.

39. Jeffrey S. Bonner; Louise Lantier; Kyle M. Hocking; Li Kang; Mark Owolabi; Freyja D. James; Deanna P. Bracy; Colleen M. Brophy; David H. Wasserman; Relaxin Treatment Reverses Insulin Resistance in Mice Fed a High-Fat Diet. Diabetes 2013, 62, 3251-3260, $10.2337 / \mathrm{db} 13-0033$.

40. Li Kang; Louise Lantier; Arion Kennedy; Jeffrey S. Bonner; Wesley H. Mayes; Deanna P. Bracy; Louis H. Bookbinder; Alyssa H. Hasty; Curtis B. Thompson; David H. Wasserman; et al. Hyaluronan Accumulates With High-Fat Feeding and Contributes to Insulin Resistance. Diabetes 2013, 62, 1888-1896, 10.2337/db12-1502.

41. Paul J. Meakin; Vicky Morrison; Claire C. Sneddon; Terhi Savinko; Liisa Uotila; Susan M. Jalicy; Jennie L. Gabriel; Li Kang; Michael Lj Ashford; Susanna Carola Fagerholm; et al. Mice Lacking beta2-Integrin Function Remain Glucose Tolerant in Spite of Insulin Resistance, Neutrophil Infiltration and Inflammation. PLOS ONE 2015, 10, e0138872, 10.1371/journal.pone.0138872. 
42. Li Kang; Julio E. Ayala; Robert Lee; Zhonghua Zhang; Freyja D. James; P. Darrell Neufer; Ambra Pozzi; Mary M. Zutter; David H. Wasserman; Diet-Induced Muscle Insulin Resistance Is Associated With Extracellular Matrix Remodeling and Interaction With Integrin $\alpha 2 \beta 1$ in Mice. Diabetes 2011, 60, 416-426, 10.2337/db10-1116.

43. Ponta, H.; Sherman, L.; Herrlich, P.A; CD44: from adhesion molecules to signalling regulators. Nat Rev Mol Cell Biol 2003, 4, 33-45.

44. Kodama, K.; Horikoshi, M.; Toda, K.; Yamada, S.; Hara, K.; Irie, J.; Sirota, M.; Morgan, A.A.; Chen, R.; Ohtsu, H., et al; et al. Expression-based genome-wide association study links the receptor CD44 in adipose tissue with type 2 diabetes. Proc Natl Acad Sci USA 2012, 109, 7049-7054.

45. Hasib, A.; Hennayake, C.K.; Bracy, D.P.; Bugler-Lamb, A.R.; Lantier, L.; Khan, F.; Ashford, M.L.J.; McCrimmon, R.J.; Wasserman, D.H.; Kang, L; et al. CD44 contributes to hyaluronan-mediated insulin resistance in skeletal muscle of high-fat-fed C57BL/6 mice. Am J Physiol Endocrinol Metab 2019, 317, E973-E983.

46. Gullberg, D.; Ekblom, P; Extracellular matrix and its receptors during development. Int J Dev Biol 1995, 39, 845-854.

47. Moore, C.J.; Winder, S.J; Dystroglycan versatility in cell adhesion: a tale of multiple motifs. Cell communication and signaling 2010 , 8,3 .

48. Jay E Brenman; Daniel S Chao; Houhui Xia; Ken Aldape; David S Bredt; Nitric oxide synthase complexed with dystrophin and absent from skeletal muscle sarcolemma in Duchenne muscular dystrophy. Cell 1995, 82, 743-752, 10.1016/0092-8674(95)90471-9.

49. Lapidos, K.A.; Kakkar, R.; McNally, E.M; The dystrophin glycoprotein complex: signaling strength and integrity for the sarcolemma. Circ Res 2004, 94, 1023-1031.

50. Mark Morgan; Martin J Humphries; Mark D. Bass; Synergistic control of cell adhesion by integrins and syndecans. Nature Reviews Molecular Cell Biology 2007, 8, 957-69, 10.1038/nrm2289.

51. Merton Bernfield; Martin Götte; Pyong Woo Park; ‡ Ofer Reizes; Marilyn L. Fitzgerald; John Lincecum; Masahiro Zako; Functions of Cell Surface Heparan Sulfate Proteoglycans. Annual Review of Biochemistry 1999, 68, 729-777,

10.1146/annurev.biochem.68.1.729.

52. Addolorata Pisconti; Jennifer D. Bernet; Bradley B. Olwin; Syndecans in skeletal muscle development, regeneration and homeostasis. Muscle Ligaments and Tendons Journal 2012, 2, 1-9.

53. Maria Elena Danoviz; Zipora Yablonka-Reuveni; Skeletal Muscle Satellite Cells: Background and Methods for Isolation and Analysis in a Primary Culture System. Breast Cancer 2012, 798, 21-52, 10.1007/978-1-61779-343-1_2.

54. Fu, X.; Wang, H.; Hu, P; Stem cell activation in skeletal muscle regeneration. Cellular and molecular life sciences 2015, 72, 16631677.

55. Gattazzo, F.; Urciuolo, A.; Bonaldo, P; Extracellular matrix: a dynamic microenvironment for stem cell niche. Biochimica et Biophysica Acta (BBA)-General Subjects 2014, 1840, 2506-2519.

56. Thomas, K.; Engler, A.J.; Meyer, G.A; Extracellular matrix regulation in the muscle satellite cell niche. Connect Tissue Res 2015, 56, $1-8$.

57. Francesca Gattazzo; Anna Urciuolo; Paolo Bonaldo; Extracellular matrix: a dynamic microenvironment for stem cell niche. Biochimica et Biophysica Acta (BBA) - Molecular Cell Research 2014, 1840, 2506-2519, 10.1016/j.bbagen.2014.01.010.

\section{Keywords}

Extracellular matrix; skeletal muscle; collagen; integrin; laminin

(C) 2020 by the author(s). Distribute under a Creative Commans CC BY license 